\title{
Synthesis, Characterization and Anti-Cancer Therapeutic Potential of Withanolide-A with 20nm sAuNPs Conjugates Against SKBR3 Breast Cancer Cell Line
}

This article was published in the following Dove Press journal:

International Journal of Nanomedicine

\author{
Qudsia Tabassam' \\ Tahir Mehmood (D) ${ }^{1,2}$ \\ Abdul Rauf Razal \\ Azmat Ullah iD $^{3}$ \\ Farhan Saeed ${ }^{4}$ \\ Faqir Muhammad Anjum ${ }^{5}$ \\ 'Department of Chemistry, University of \\ Sargodha, Sargodha, Pakistan; ${ }^{2}$ Institute \\ of Biochemistry and Biotechnology, \\ University of Veterinary and Animal \\ Sciences-UVAS, Lahore, Pakistan; \\ ${ }^{3}$ Department of Food Science and \\ Human Nutrition, University of \\ Veterinary and Animal Sciences-UVAS, \\ Lahore, Pakistan; ${ }^{4}$ Institute of Home \& \\ Food Sciences, Government College \\ University Faisalabad, Faisalabad, \\ Pakistan; ${ }^{5}$ Vice Chancellor Secretariat, \\ University of the Gambia, Banjul, The \\ Gambia
}

\begin{abstract}
Background: Nanotechnology is gaining emerging interest in advanced drug discovery therapeutics due to their tremendous properties including enhanced delivery of therapeutic payload, extensive surface to volume ratio, high permeability, retention behaviors, etc. The gold nanoparticles (AuNPs) are favored due to their advanced features, such as biogenic, tunable physiochemical response, ease in synthesis, and wide range of biomedical applications. The phytochemicals have been focused to design Au nano-carrier-based conjugation for active-targeting drug delivery due to their nano conjugation ability.
\end{abstract}

Aim: The present study describes the facile synthesis of $20 \mathrm{~nm}$ spherical AuNPs and their conjugation with reported anti-cancer phytocompound Withanolide-A (1).

Methods: The $20 \mathrm{~nm}$ sAuNPs were synthesized chemically and characterized their phytochemical gold nanoconjugates through UV-visible spectroscopy, dynamic light scattering (DLS) and transmission electron microscopy (TEM) imaging techniques. The anti-cancer therapeutic potentials were tested with both nanoconjugates and pure WithanolideA (1) by using SKBR3 breast cancer cells line.

Results: The synthesized sAuNPs showed significant conjugation with Withanolide-A and showed stability. Furthermore, these Au nanoconjugates with Withanolide-A (1) significantly induce blockage of SKBR3 cell growth at half maximal active concentration that compared to pure Withanolide-A (1).

Conclusion: Our findings provide a foundation to further progress how they can overcome cancer drug resistance by conjugating active drugs in combination with AuNPs through optimizing the effective drug concentration and removing the surface barrier.

Keywords: Withania somnifera, Withanolide-A, gold nanoparticle; AuNP, breast cancer, anti-cancer and incucyte

\section{Introduction}

Nanotechnology has received a lot of attention in recent years because of its enhanced payload to targeted therapeutics sites, modifying cellular permeability, uptake or pharmacokinetic profile. ${ }^{1-3}$ Among the diverse range of nanoparticle carriers (iron oxide, silicone material and quantum dots) the AuNPs are favored due to their high biocompatibility, quenching efficiencies, facile synthesis, multiple functionality and tunable optical nature. ${ }^{4-7}$ Several studies $^{8-11}$ reported a mechanism of action of AuNPs as an active carrier of drug payload to targeted
Correspondence: Tahir Mehmood; Faqir Muhammad Anjum

Email tahir.mehmoodbiochem@uvas.edu.pk; dranjum@utg.edu.gm 
site. Their interaction modifies the cell and surface protein interaction kinetics profile; however, their action varies with size and charge factor. The previously reported study describes through ultra-high-resolution imaging atomic force revealed that AuNPs carrier have tendency to alter membrane roughness of cell, which enhanced their cellular uptakes. $^{12}$

Breast cancer is frequently diagnosed among women worldwide, with the highest rate in Australian women, diagnosed more than other types of cancer. ${ }^{13,14}$ The accelerated rate of mortalities of around $73 \%$ in the metastatic disease is usually linked with various genetic and epigenetic factors. ${ }^{13,15,16}$ The conventional therapies have a number of flaws: poor accessibility to tumor tissues, nonselectivity, severe side-effects - hair loss, diarrhea, high dose, less efficacy, and safety profile concerns - which always demand novel and more effective treatment methods for this type of cancer. In this context, we prepared AuNPs combinational strategy with a reported anticancer compound (Withanolide-A) is being using to overcome disease burden and more effective potential. ${ }^{17}$

The phytochemical-based remedies have been reported over thousands of years ago to treat multiple disorders in traditional medicinal system and many of them approved by Food and Drug Administration (FDA) clinically. ${ }^{18}$ In previous years Withania somnifera extract passed clinical trial phase one (ID number: NCT00817752) against cancer disorders in 2010 and powder extracts passed phase and second clinical trial (ID number: NCT00689195) against osteosarcoma in 2011. The withanolide compounds from the medicinal plant Withania somnifera have been extracted and tested in vitro and in vivo against a number of disorders; including as an anticancer, neuromodulators, antidiabetic, adaptogenic, cardioprotective, and neuroprotective agent, etc. ${ }^{19-21}$

However, there are numbers of issues raised related to the use of Withanolide-A (1), so previous raised issues should be addressed; either concern with their low solubility or lesser bioavailability (rapid passage to type I, II type metabolism) and improved pharmacokinetics profile with enhanced targeted cellular uptake and delivery, which may modify its unique functions. Figure 1 showed the structural formula of tested compound 1.

The application of nanobiotechnology along with the combination therapies is discussed in a number of studies, where nanoparticles conjugate with phytochemical for enhanced drug delivery mechanismresearchers. ${ }^{22-25}$ The current study was designed to synthesize 20nm sAuNPs and their conjugation with the previously reported anticancer

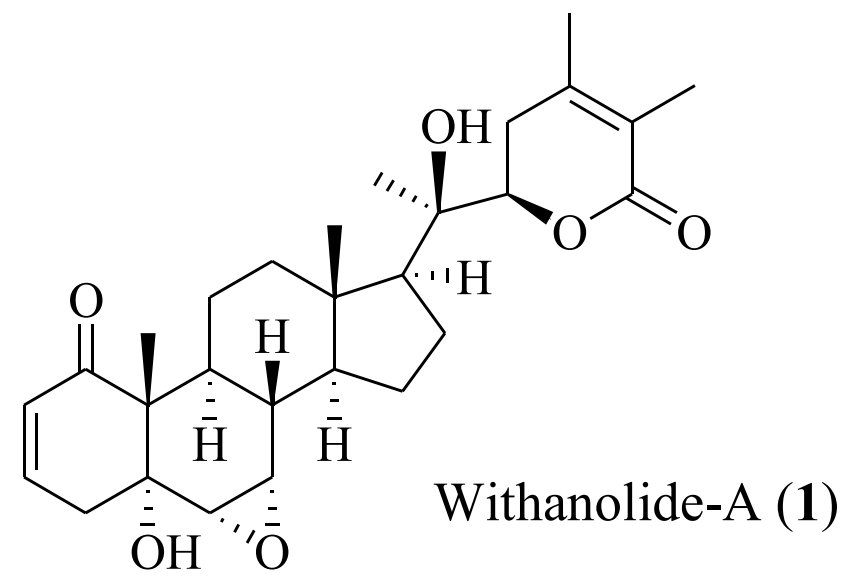

Figure I Structural formula of Withanolide-A (I). A phytocompound from Withania somnifera.

compound Withanolide-A (1), ${ }^{26,27}$ which was extracted from the Withania somnifera plant and purified to test cytotoxicity against the SK-BR3 breast cancer cells line. To the best of our knowledge only limited work has evaluated this before, so we believe this is first time there has been a comprehensive study of $20 \mathrm{~nm}$ sAuNPs +1 conjugation and cytotoxicity study against the breast cancer SKBR3 cell line.

\section{Experimental Design Chemical Materials}

The Withanolide-A (1) (CAS: 32911-62-9) was purchased from ChromaDex. $\mathrm{HAuCl}_{4} \cdot 3 \mathrm{H}_{2} \mathrm{O}$ (99.99\%); tri-sodium citrate $\left(\mathrm{Na}_{3}\right.$ citrate), tannic acid and $\mathrm{K}_{2} \mathrm{CO}_{3}$ (Potassium carbonate) were purchased from Sigma-Aldrich (Australia). The phosphate buffer saline (PBS) was procured from Gymea, NSW, Australia. Formvar/carbon coated, square 200 mesh size copper grids and MillexGV syringe filters $(0.22 \mu \mathrm{m}$, PVDF, Cat. No. SLGV033RS) were purchased from Sigma-Aldrich (Australia). The RPMIM (Roswell Park Memorial Institute Medium), MTT reagent and DMSO $\left(\mathrm{Me}_{2} \mathrm{SO}\right)$ were purchased from Life Sci. Technology, Australia. The deionized $\mathrm{H}_{2} \mathrm{O}$ (electric resistivity $>18 \mathrm{M} \Omega . \mathrm{cm}$ ) was supplied by Milli-Q.

\section{Cell Culture}

The SKBR-3 cell line was a kind gift from Dr. Thomas Grewal at the University of Sydney. The cell line procured from the American Type Culture Collection (ATCC). The Cell line was authenticated via short tandem repeat (STR) polymorphism, fingerprint analyses and single nucleotide 
polymorphism (SNP), passaged for less than 6 months, and cultured as formerly described. ${ }^{28}$

\section{Instrument Used}

The UV-Vis spectrophotometer (Shimadzu 2600, Japan), Ultracentrifugation Sorvall $^{\mathrm{TM}}$ WX Floor (Thermo scientific USA), DLS (Nano ZS, Malvern Instruments, UK), transmission electron microscopy (TEM; JEOL-JEM-1400, Tokyo, Japan), Incucyte, Multi-plate reader (Perkin-Elmer victor X4, US), Incucyte (Incucyte ${ }^{\circledR}$ ZOOM Live-cell Analysis System ESSEN, UK) were used during study.

\section{Synthesis of Multifunctional and Spherical 20nm Au Nanoparticles (sAuNPs) and Conjugation with Withanolide-A (I) Phytochemical \\ Preparation of Different Working Solutions}

The $\mathrm{AuCl}_{3}$ solution (12.5 mM or $0.05 \%$ ) was prepared by the addition of $49.2 \mathrm{mg}$ of $\mathrm{HAuCl}_{4} \cdot 3 \mathrm{H}_{2} \mathrm{O}$ in sterile Milli-Q $\mathrm{H}_{2} \mathrm{O}(10 \mathrm{~mL})$. The $\mathrm{Na}_{3}$ citrate $(1 \% \mathrm{TSC})$ solution was prepared by dissolving $100 \mathrm{mg}$ of $\mathrm{Na}_{3}$ citrate in sterile Milli-Q $\mathrm{H}_{2} \mathrm{O}(10 \mathrm{~mL})$. A $2.5 \mathrm{mM}$ tannic acid (TA) solution was prepared by dissolving $21.2 \mathrm{mg}$ of it in $1 \% \mathrm{TSC}$ solution $(5 \mathrm{~mL})$. The aq. $\mathrm{K}_{2} \mathrm{CO}_{3}$ solution $(150 \mathrm{mM})$ was prepared by dissolving of $103.6 \mathrm{mg}$ anhydrous $\mathrm{K}_{2} \mathrm{CO}_{3}$ in sterile Milli-Q $\mathrm{H}_{2} \mathrm{O}(5 \mathrm{~mL})$. A $10 \mathrm{mM}$ or $1 \times$ PBS storage buffer ( $\mathrm{pH}$ 7.4) was prepared by dissolving 2 tablets in $200 \mathrm{~mL}$ of sterile Milli-Q $\mathrm{H}_{2} \mathrm{O}$ and was filtered through $0.22 \mu \mathrm{m}$ filter paper to obtain a $10 \mathrm{mM}$ or $1 \times$ PBS phosphate buffer solution ( $\mathrm{pH} 7.4$ at $\left.25^{\circ} \mathrm{C}\right)$.

\section{Colloidal sAuNPs Synthesis and Conjugation with Withanolide-A (I)}

The synthesis of $20 \mathrm{~nm}$ colloidal mono-dispersed sAuNPs solution was prepared following a protocol reported by Turkevich et al (2006), Frens et al $(1973)^{29,30}$ and Jordi Piella et al (2016). ${ }^{31}$ The $12.5 \mathrm{mM}(0.05 \%) \mathrm{AuCl}_{3}$ solution in Milli-Q $\mathrm{H}_{2} \mathrm{O}, 1 \%$ TSC in Milli-Q $\mathrm{H}_{2} \mathrm{O}, 2.5 \mathrm{mM}$ TA dissolved in $1 \%$ TSC and $150 \mathrm{mM}$ aq. $\mathrm{K}_{2} \mathrm{CO}_{3}$ in Milli-Q water were prepared. The $0.05 \% \mathrm{AuCl}_{3}$ solution $(0.6 \mathrm{~mL})$ was diluted to $25 \mathrm{~mL}$ with Milli-Q $\mathrm{H}_{2} \mathrm{O}$ and stirred for two minutes. Then, 1\% TSC $(150 \mu \mathrm{L}), 2.5 \mathrm{mM}$ TA $(40 \mu \mathrm{L})$ and $150 \mathrm{mM}$ aq. $\mathrm{K}_{2} \mathrm{CO}_{3}(40 \mu \mathrm{L})$ were added into a previously mixed solution and stirred slowly for 5 minutes at room temperature. The color of the mixed solution changed from light yellow to ash black. The wrapped glass container was charged with magnetic bar at $60{ }^{\circ} \mathrm{C}$ for 5 minutes without stirring. The mixture changed the color from dark to wine red and finally purple within 1-2 minutes due to presence of high reacting citrate-tannic acid complex. The heated Au nanoparticles colloidal solution was kept at $40{ }^{\circ} \mathrm{C}$ for 10 minutes followed by cooling to ambient temperature and finally stored at $4{ }^{\circ} \mathrm{C}$. After cooling, the prepared sample was diluted around 1.0 OD (Optical Density) value. ${ }^{32}$ For conjugation, $10 \mu \mathrm{g}$ of Withanolide-A (1) was dissolved in $1 \mathrm{~mL}$ of sAuNPs solution followed by overnight incubation in cold room with gentle shaking. For stability analysis, $10 \mu \mathrm{L}$ of $10 \% \mathrm{NaCl}$ solution was added into conjugated and control samples for 15 minutes to check the color change as well as UV/Vis, TEM and Zetasizer measurements. ${ }^{33}$

\section{Conjugation Purification}

The number of moles in $1 \mathrm{~mL}$ gold solution was calculated and various molar excess compounds were tried to cover the gold particle surface. Interestingly, we found a nice surface covering with $10 \mu \mathrm{g} / \mathrm{mL}$ compound concentration that was checked with UV shift. There was no absorption of compound detected in supernatant. All the compounds might be bound to nanoparticles according to reports by Farooq et al (2018). ${ }^{1}$ Abs of conjugated compoundunconjugated compound in supernatant/Abs conjugated compound*100.

\section{Characterization of Bare sAuNPs and Phyto-Conjugates UV-Vis Spectroscopy}

To obtain absorption spectra phyto-conjugate $(70 \mu \mathrm{L}$ of bare sAuNPs) solution and Withanolide-A (1), the solution was measured from 200 to $800 \mathrm{~nm}$ with blank subtraction of media and distil $\mathrm{H}_{2} \mathrm{O}$.

\section{Dynamic Light Scattering (DLS)}

Certain physical parameters of conjugated nanoparticle such as polydispersity index (PDI), average particle hydrodynamic diameter and surface charge were measured with DLS. The sample was diluted to1:10 and 1:2000 with Milli-Q $\mathrm{H}_{2} \mathrm{O}$ for size and zeta-potential analysis respectively. All the measurements were done in triplicate and reported as average of \pm standard deviation (SD).

\section{Transmission Electron Microscopy (TEM)}

To check the particle core image before and after conjugation, TEM analysis was performed by using small metal 


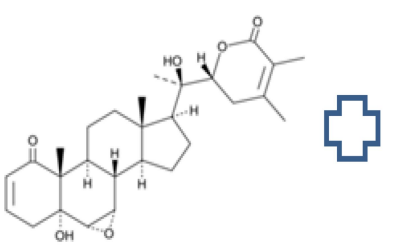

Withanolide A

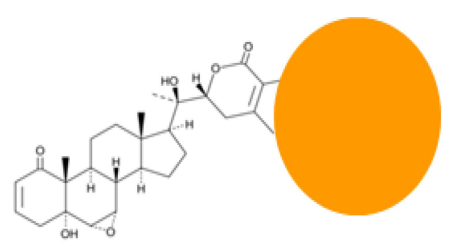

Withanolide A-sAuNP

Figure 2 Conjugation of Withanolide-A with 20nm sAuNPs.

grid. Briefly, $10 \mu \mathrm{L}$ of 1:4 diluted sample solution with and without conjugation were applied on $\mathrm{Cu}$ grid having C coating. Next, the grid was air dried overnight and analysis was performed at $120 \mathrm{kv}$.

\section{Cell Culture and Cytotoxic Evaluation}

The SKBR3 breast cancer cells were grown into RPMI media that was supplemented with $10 \%$ fetal bovine serum (FBS) and 1\% suspension of penicillin-streptomycin. The flask mixture could grow under standard condition in incubator, which was humidified with $5.0 \% \mathrm{CO}_{2}$ level at $37{ }^{\circ} \mathrm{C}$. To check the Au-phytoconjugates cytotoxicity, 5000 cells/well were seeded for 24 hours. The next day's cells were treated into collagen-coated Thermanox plastic well with corresponding concentrations of the tested sample $10,20,40 \mu \mathrm{g} / \mathrm{mL}$ and replenished with fresh media containing negative DMSO $1 \%$ and AuNPs $40 \mu \mathrm{L} / \mathrm{mL}$ with positive control $10 \mu \mathrm{g} / \mathrm{mL}$ (Cisplatin drug) in triplicates. After specific time intervals $(0,24,48$, and $72 \mathrm{~h}$ ) image collection of the well was done with $10 \times$ magnification using Incucyte zoom. After 72 hours, the percentage of confluence/time was obtained by using v2016A incucyte software to generate cell growth curves over time. The cell viability assay was performed by using MTT reagent $(20 \mu \mathrm{L} / 100 \mathrm{~mL})$ and cell viability count was done according to following equation:

$\%$ cell viability $=($ Average of treated cell absorbance-BLANK/Average of non-treated cell absorbance-BLANK) $\times 100$
Where, blank is MTT reagent and non-treated cells are negative control such as media and AuNPs correspond concentration in media. ${ }^{34}$

\section{Statistical Analysis}

All the experiment was performed in triplicate to obtained the mean value and SD. For data processing SPSS software 19 and Microsoft Excel were used. The difference in activities of various group in term of mean was checked by Tukey post-hoc analysis where $p<0.05$ is an indication of statistically significant. ${ }^{3}$

\section{Results and Discussion}

\section{Synthesis of Au-Phyto-Nanconjugate and}

\section{Characterization}

The AuNPs can be synthesized by various in vitro and in situ methods but uniform spherical particle only acquired by limited strategies. For ease of method working procedure and apparatus used chemical reduction considered as one of favorite method for synthesis of these spherical AuNPs synthesis, which involves reduction and stabilization. In this study, chemical synthesis method was used to prepare spherical AuNPS. It is already known that phytochemical withanolides possess strong hydrophobic hydrogen and electrostatic interactions. Herein, we conjugate Withanolide-A (1) $10 \mu \mathrm{g} / \mathrm{mL}$ with spherical AuNP solution according to the same strategy as outlined in ${ }^{26}$ Figure 2.

After conjugation the phytonanoconjugte of sAuNP+1 subjected to ultracentrifugation to obtained purified red

Table I Phyto-Nanoconjugate Size, Zeta Potential and UV-Absorption Maximum and TEM Characterization

\begin{tabular}{|l|l|l|l|l|l|}
\hline Sample & Z-Avg Size (nm) & Polydispersity Index (PDI) & Zeta Potential (mV) & $\lambda \mathbf{m a x}(\mathbf{n m})$ & TEM (nm) \\
\hline Bare sAuNP & $25.35 \pm 0.610$ & $0.285 \pm 0.020$ & $-44.3 \pm 0.860$ & 523.5 & 20 \\
Phyto-nanoconjugate AuNPs & $29.73 \pm 0.650$ & $0.3 \pm 0.009$ & $-20 \pm 0.100$ & 525.5 & 20 \\
\hline
\end{tabular}

Note: Values are means $\pm S D(n=3)$ of three separate experiments.

Abbreviations: TEM, transmission electron microscopy; UV, ultraviolet. 
loose pellets for $40 \mathrm{~min}$ at $17,000 \mathrm{rpm}$ at $10^{\circ} \mathrm{C}$. The pellet was used to perform further analysis.

\section{UV-Vis}

The UV-Vis spectroscopy is a sensitive method for detecting colloidal metal, due to exhibiting a characteristic absorption peak at about $490-600 \mathrm{~nm}$, which is attributed to surface plasmon excitation. The absorption band maximum for bare sAuNPs and phytoconjugates were observed at $\lambda_{\max } 523.5$ and $525.5 \mathrm{~nm}$ respectively (a little bit shift is mentioned in Table 1 and shown in Figure 3) (supplemental Figure 1).

These shifts indicate the formation of adsorbed layers around AuNPs. Aggregation of AuNPs is accompanied

\section{UV Absorbance}

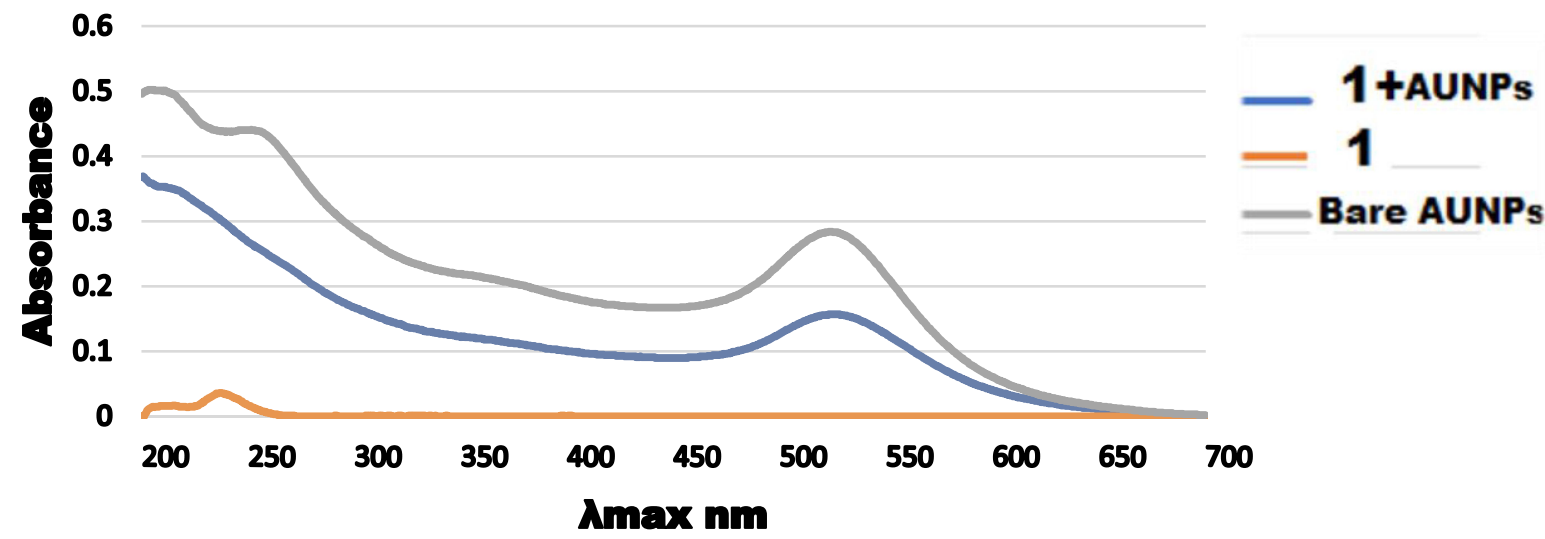

A

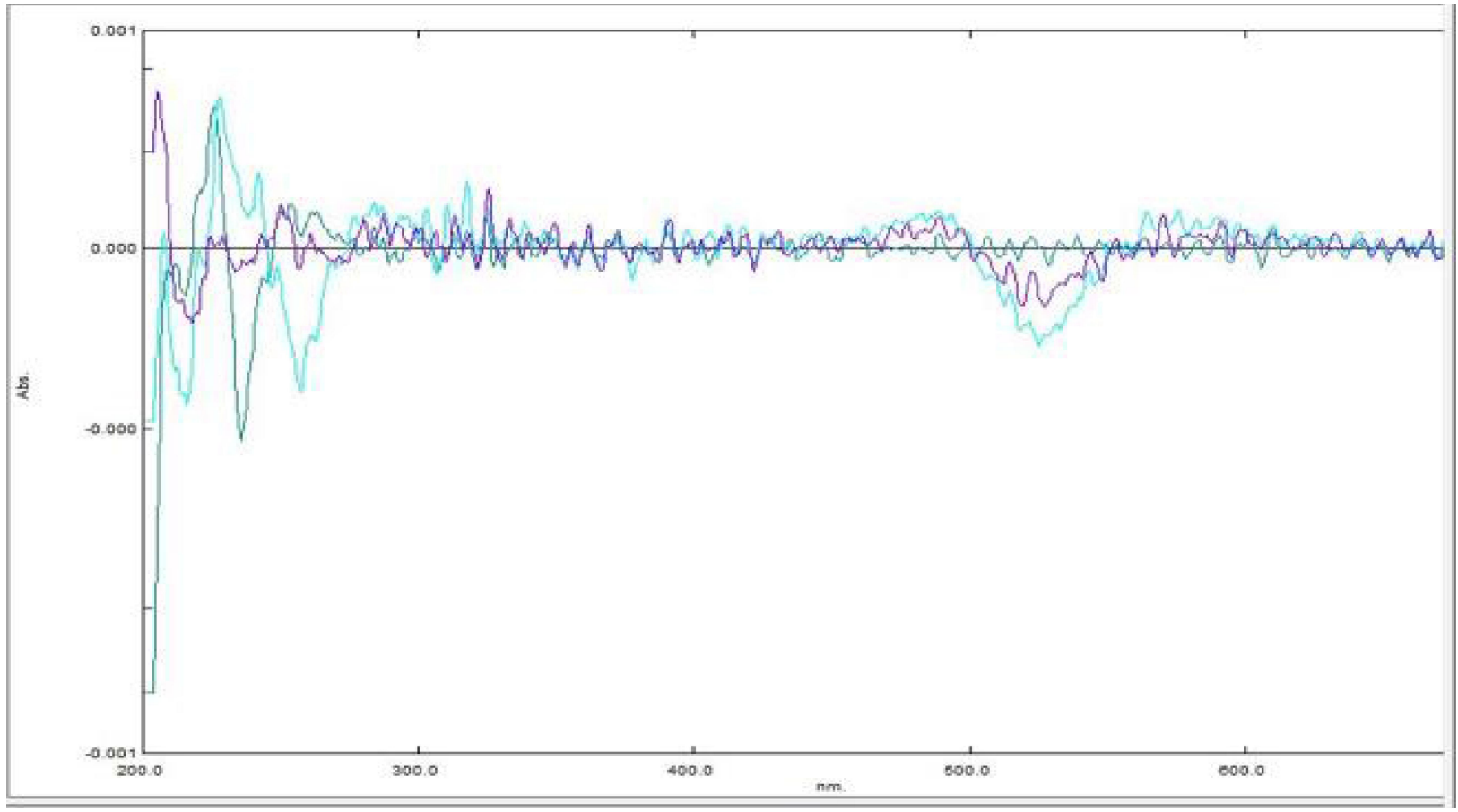

B

Figure 3 Image (A) UV absorbance of $20 \mathrm{~nm}$ Bare spherical Au nanoparticles (sAuNPs), Withanolide-A (I) and I+sAuNPs. Image (B). Secondary derivative green line corresponds to compound $\mathbf{I}$ absorbance followed by blue line Au nanoparticles and purple $\mathbf{I}+$ sAuNPs absorbance. 


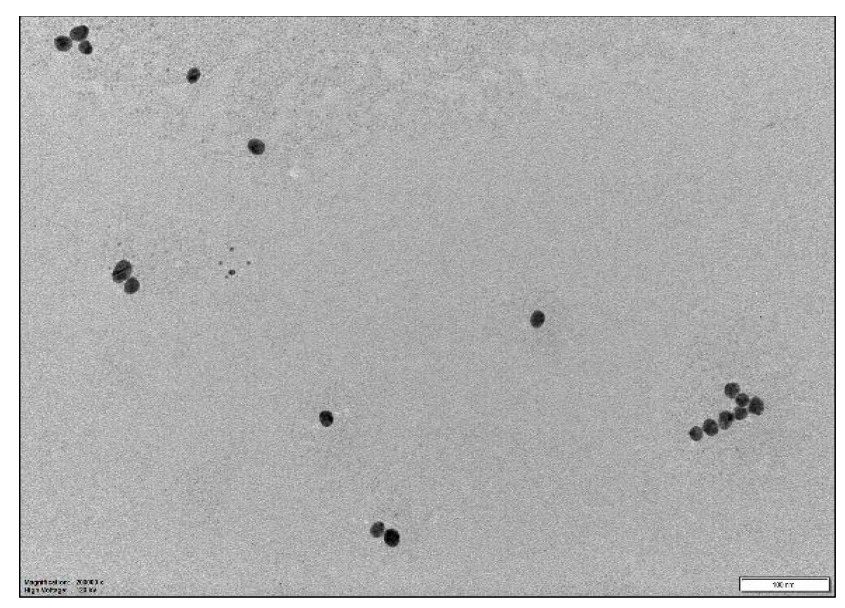

A

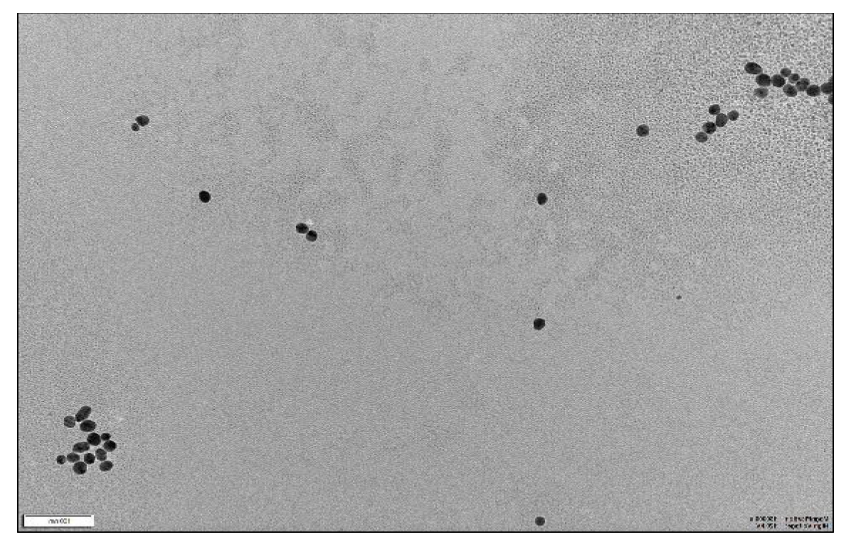

B

Figure 4 Image $(\mathbf{A})$ is for Bare spherical Au nanoparticles and image (B) is for spherical Au nanoparticles + Withanolide-A at $100 \mathrm{~nm}$ scale bar. with a change of color: optical absorption spectroscopy quantifies this process. We did not noticed any color change in our tested samples before and after conjugation, which is similar reliability parameter as reported in previous finding. ${ }^{35,36}$ These sAuNPs showed $\lambda_{\max }$ at 523.5 $\mathrm{nm}$, which is quite close in agreement with Shafiq et al $(2018)^{37}$ work who reported that $20 \mathrm{~nm}$ particle is for $\lambda_{\max }$ $522 \mathrm{~nm}$. Furthermore, secondary derivatives were recorded which ensure us confidence on binding between two moieties with stability Figure 3.

\section{Dynamic Laser Scattering DLS}

Characteristic features, such as hydrodynamic size; PDI and surface charge were measured with DLS before and after conjugation with average corresponding value (Table 1). Before and after conjugation, Zeta potential value showed from $-44.3 \pm 0.86 \mathrm{mV}$ to $-20 \pm 0.1 \mathrm{mV}$, which was a significant difference due to the presence of a slightly positively charged hydrogen atom of 1 on the Au surface. The PDI changed a little bit from $0.285 \pm 0.02$ to $0.3 \pm 0.009$, which was less than 0.3 . It indicates that the particles were not aggregated and homogenous in size hydrodynamic size was less increased from $25.35 \pm 0.61 \mathrm{~nm}$ to $29.73 \pm 0.65 \mathrm{~nm}$ and authenticates that 1 molecule attached on $\mathrm{Au}$ surface. ${ }^{13}$ The results are presented in Table 1 , before and after conjugation showed stability of this nanoconjugate. (See detail Supplemental Figure 2).

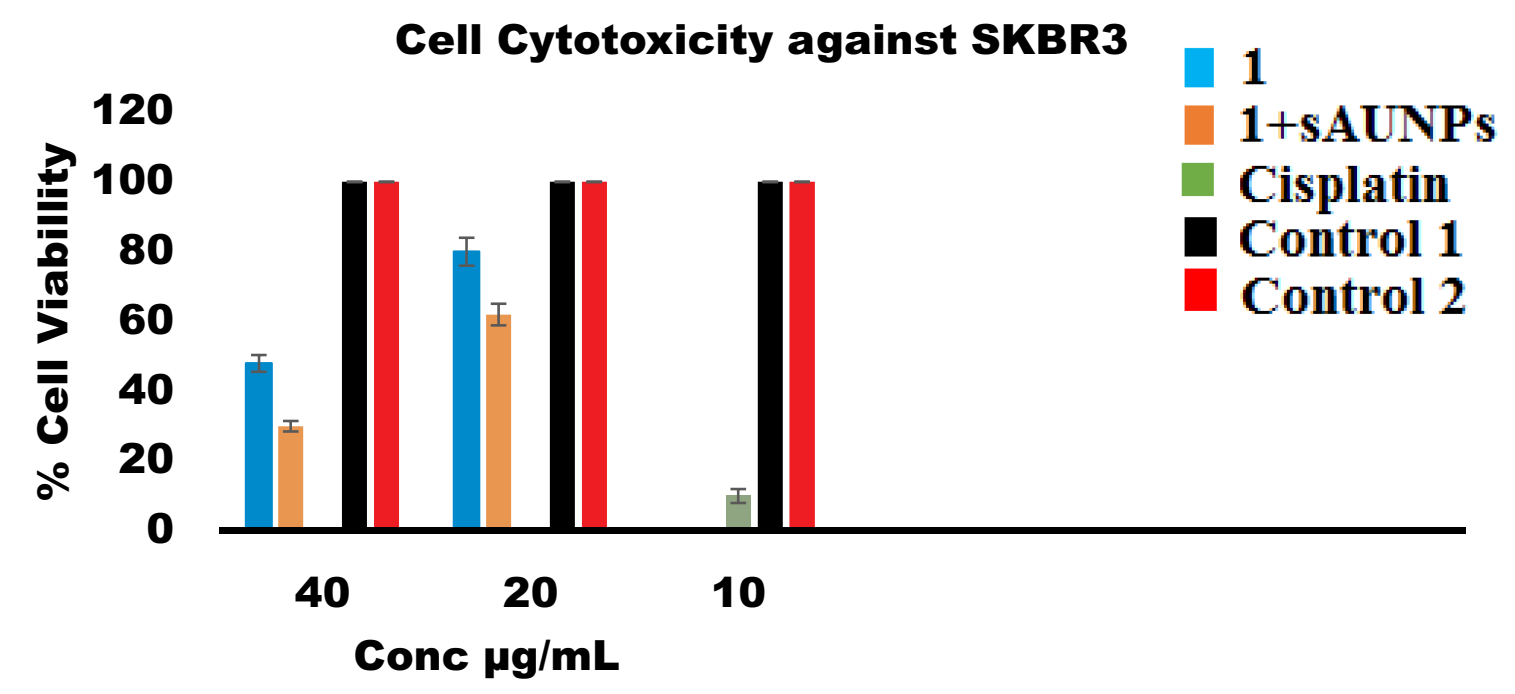

Figure 5 Anticancer activity of Withanolide-A, Phyto-nanoconjugate at different concentration against SKBR3 Cell line. While control I and 2 correspond to AuNPs and media with corresponding concentration of DMSO ( $\%$ used to prepared sample Withanolide-A). Values indicate mean \pm SD of three independent experiment. While, activity-based differences among various concentration found significant having $p<0.05$. 


\section{Transmission Electron Microscopy (TEM)}

Characterization of bare and conjugated nano formulation through TEM displayed a uniform structural morphology and distribution. The size and size distribution of synthesized AuNPs were determined using TEM Figure 4. The average size $20 \mathrm{~nm}$ was recorded on micrograph reported in (Table 1) before and after conjugation (see more images in supplemental Figure 3).

\section{Determination of Cytotoxicity}

We compared free compound 1 anticancer activity and phytonanconjugate with $20 \mathrm{~nm}$ sAuNPs against SKBR3 breast cancer cell lines. The standard MTT assay ${ }^{38}$ were used to determine the level of cell viability coupled with $\%$ confluence analysis of incucyte $10 \times$ magnification images results software generated growth curve/time. ${ }^{39}$ The anticancer compound 1 with its varying concentration 40, 20, $10 \mu \mathrm{g} / \mathrm{mL}$ along with the same nanoconjugate concentration applied as triplicate.

In the MTT assay results a dose dependent cytotoxicity response was observed. Interestingly, we found that our phytonanoconjugate shows higher antiproliferative effects and showed cell viability of $30 \%$ compared to 1 alone of $45 \%$ at the concentration of $40 \mu \mathrm{g} / \mathrm{mL}$ followed by $60 \%$ and $80 \%$ at $20 \mu \mathrm{g} / \mathrm{mL}$, which might be due to higher uptake of complex mixture in nucleus or cytoplasm functionalized Au nanoparticle carrier. The results followed the same behavior towards cell viability a we observed in Vijayakumar $(2019)^{40}$ studies, the Au nanoconjugate showed a higher antiproliferative profile than with conjugation.

As has already been proven in several studies (see, for example, Agarwalla et al, 2016; ${ }^{41}$ Wing Lee et al, 2016; ${ }^{13}$ and Lara et al, 2019), ${ }^{8}$ Au nanoparticle conjugated with phytochemical enhanced their payload and cellular uptake. However, in our findings we noticed slightly higher cell growth inhibition activity at concentration $40 \mu \mathrm{g} / \mathrm{mL}$ with 1 and $20 \mu \mathrm{g} / \mathrm{mL}$ in Au nanoconjugate sAuNP+1 compared to previous literature as shown in Figure 5 (supplemental Figure 4). There was no cell growth inhibition observed with corresponding negative control, while standard cisplatin drug behaves better than 1 and phytonanconjugate.

A similar trend has been seen with incucyte studies result where a minimum percentage of live cell confluency (number of cells) observed with Cisplatin $(10 \mu \mathrm{g} / \mathrm{mL})>$ $(40 \mu \mathrm{g} / \mathrm{mL} 1+20 \mathrm{~nm} \mathrm{sAuNPs})>(40 \mu \mathrm{g} / \mathrm{mL} 1)>(20 \mu \mathrm{g} /$ $\mathrm{mL} 1+20 \mathrm{~nm} \mathrm{sAuNPs})>(20 \mu \mathrm{g} / \mathrm{mL} 1)>(10 \mu \mathrm{g} / \mathrm{mL} 1+$ $20 \mathrm{~nm}$ sAuNPs $)>(10 \mu \mathrm{g} / \mathrm{mL} 1)>$ Media $>$ AuNPs (Figures 6 and 7 (A, B).

Due to the higher uptake and active carrier delivery through AuNPs based targeted drug delivery it is possible to destroy the cancer cells by combining phytochemical with minimal effective cytotoxic concentration due to higher safety profile. Therefore, it is worthwhile exploring the biosynthesized nanoparticles as a possible source of novel anticancer drugs.

\section{Conclusion}

In conclusion this study has reported the effective synthesis of $20 \mathrm{~nm}$ sAuNPs and their conjugation with phytochemical anticancer compound 1, which was previously reported safe to normal cell line. Later characterization was done with UV-Vis, DLS and TEM techniques. Three important points observed as valuable findings. The synthesized phytonanconjugate proved as a stable moiety,

\section{Cytotoxicity}

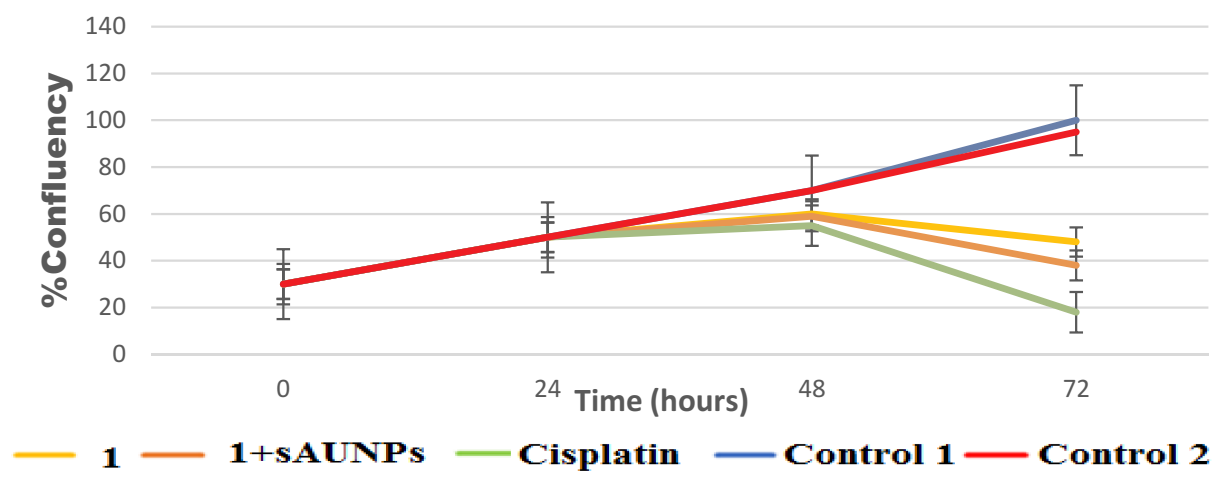

Figure 6 Percentage of Confluency (number of cell) growth curve/time against SKBR3 breast cancer cell line. There was significant difference found with specific interval of time having $p<0.05$. 


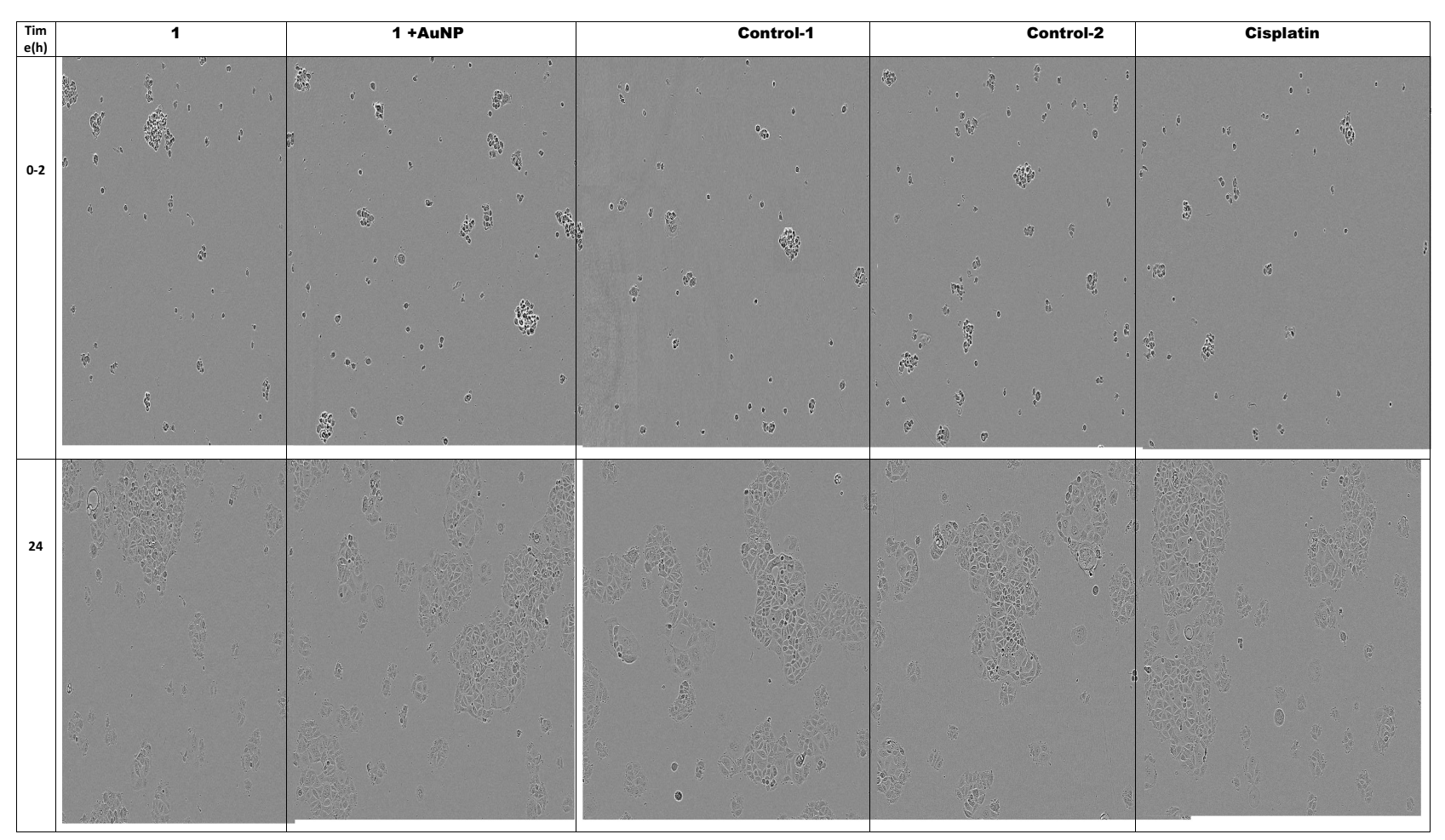

A

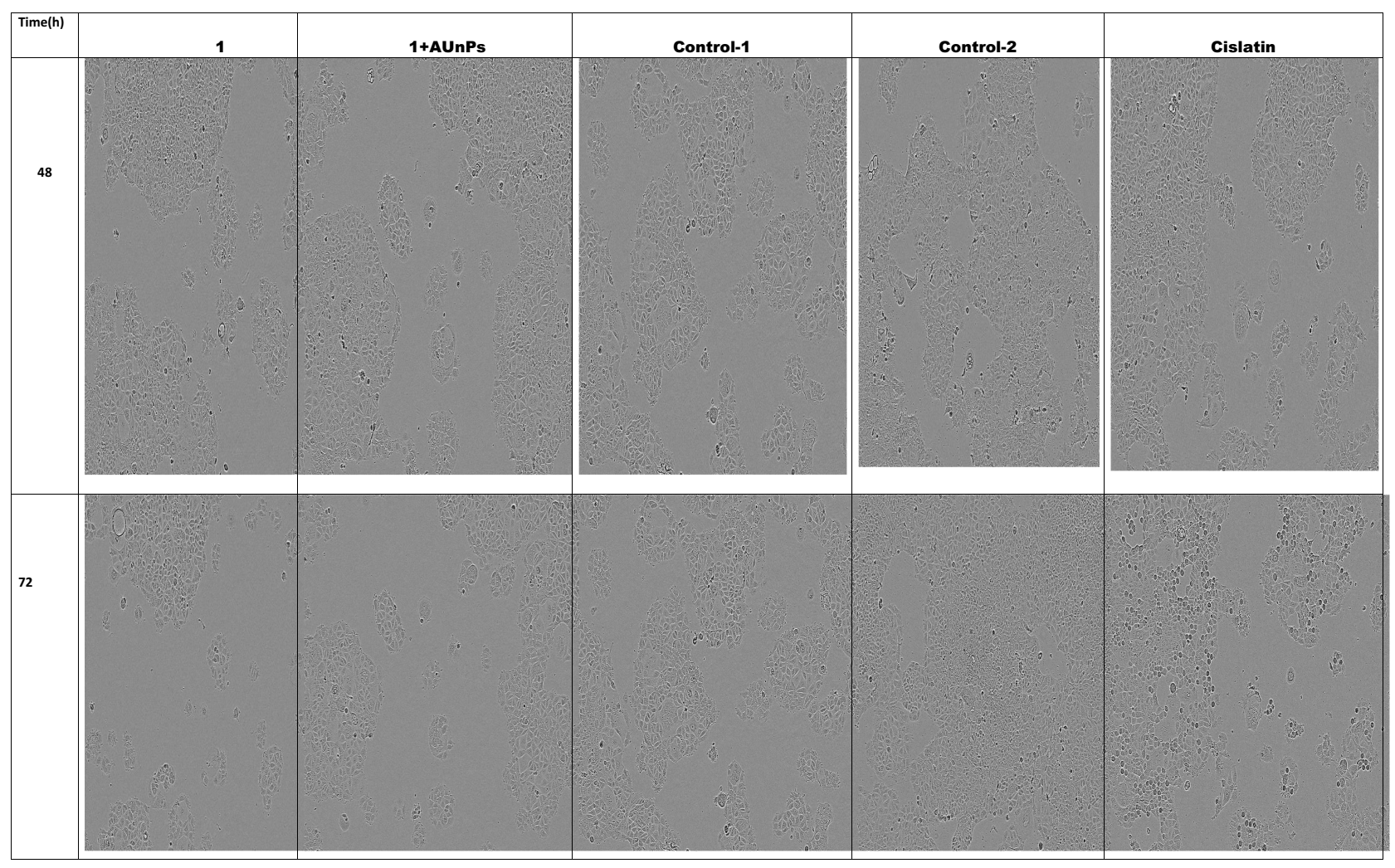

B

Figure 7 Image collection of cell growth inhibition assay after specific interval of time. (A) Images at time 0-2 (h). (B) Images at time 48.72(h). 
tested nanoconjugate showed higher toxicity than normal compound and values of cytotoxic concentration seem to be higher. However, the stability and better performance of these phytonanconjugate towards in vitro SKBR3 cells make it an active target to explore for other applications in future.

\section{Abbreviations}

Withanolide-A, ATCC, American Type Culture Collection; $\mathrm{Au}$, gold; AuNPs, gold nanoparticles; DLS, dynamic light scattering; DLS, dynamic light scattering; DMSO, dimethyl sulfoxide; $\mathrm{HAuCl}_{4}$, chlorauric acid or tetrachloroauric acid; nm, nano meter; PBS, phosphate buffer saline; PDI, polydispersity index; PVDF, polyvinylidene fluoride; sAuNPs, spherical Au nanoparticles; sAuNPs, spherical Au nanoparticles; SKBR3, Sloan Kettering-breast cancer; STR, short tandem repeat; TA, tannic acid; TEM, transmission electron microscope; TEM, transmission electron microscopy.

\section{Acknowledgment}

We would like to thank School of Pharmacy, Madsen Building, Bosch Institute, University of Sydney (Australia) for sharing their instrument with us to complete this study. We would like thank to Dr. Veysel Kayser for funding and all support to complete this research work.

\section{Disclosure}

The authors report no conflicts of interest in this work.

\section{References}

1. Farooq MU, Novosad V, Rozhkova EA, et al. Nanoparticles-enabled efficient dual delivery of anticancer therapeutics to HeLa Cells. Sci Rep. 2018;8(1):1-12. doi:10.1038/s41598-018-21331-y

2. Patil MP, Jin X, Simeon NC, et al. Anticancer activity of Sasa borealis leaf extract-mediated gold nanoparticles. Artif Cells Nanomedi Biotechnol. 2018;46(1):82-88. doi:10.1080/21691401.2017.1293675

3. Lee WH, Loo CYHX, Ong D, Traini P, Young M, Rohanizadeh R. Synthesis and characterization of inhalable flavonoid nanoparticle for lung cancer cell targeting. J Biomed Nanotechnol. 2016;12 (2):371-386. doi:10.1166/jbn.2016.2162

4. Wilhelm S, Tavares AJ, Dai Q, et al. Analysis of nanoparticle delivery to tumours. Nat Rev Mater. 2016;1(5):1-12. doi:10.1038/natrevmats. 2016.14

5. Balakrishnan S, Bhat FA, Raja Singh P, et al. Gold nanoparticle-conjugated quercetin inhibits epithelial-mesenchymal transition, angiogenesis and invasiveness via EGFR/ VEGFR-2-mediated pathway in breast cancer. Cell Prolif. 2016;49 (6):678-697. doi:10.1111/cpr.12296

6. Kyriazi ME, Giust D, El-Sagheer AH, et al. Multiplexed mRNA sensing and combinatorial-targeted drug delivery using DNA-gold nanoparticle dimers. ACS Nano. 2018;12(4):3333-3340. doi:10.1021/ acsnano. $7 \mathrm{~b} 08620$
7. Lukianova-Hleb EY, Ren X, Sawant RR, Wu X, Torchilin VP, Lapotko DO. "On-demand intracellular amplification of chemoradiation with cancer-specific plasmonic nanobubbles. Nat Med. 2014;20 (7):778-784. doi:10.1038/nm.3484

8. Lara-Cruz C, Jiménez-Salazar JE, Arteaga M, et al. Gold nanoparticle uptake is enhanced by estradiol in MCF-7 breast cancer cells. Int J Nanomedicine. 2019;14:2705. doi:10.2147/IJN.S196683

9. Tan G, Onur MA. Cellular localization and biological effects of 20nm-gold nanoparticles. J Biomed Mater Res. 2018;106(6):17 08-1721. doi:10.1002/jbm.a.36373

10. Almeida JPM, Lin AY, Figueroa ER, Foster AE, Drezek RA. In vivo gold nanoparticle delivery of peptide vaccine induces anti-tumor immune response in prophylactic and therapeutic tumor models. Small. 2015;1(12):1453-1459. doi:10.1002/smll.201402179

11. Ryan JA, Overton KW, Speight ME, et al. Cellular uptake of gold nanoparticles passivated with bsa-sv40 large $t$ antigen conjugates. Anal Chem. 2007;79(23):9150-9159. doi:10.1021/ac0715524

12. Lee CW, Jang, Pan HJ, Chen YR, Chen CC, Lee CH, Lee C-H. Membrane roughness as a sensitive parameter reflecting the status of neuronal cells in response to chemical and nanoparticle treatments. J Nanobiotechnology. 2016;14(1). doi:10.1186/s12951-016-0161-5

13. Wang D, Naydenov NG, Dozmorov MG, Koblinski JE, Ivanov AI. Anillin regulates breast cancer cell migration, growth, and metastasis by non-canonical mechanisms involving control of cell stemness and differentiation. Breast Cancer Res. 2020;22(1). doi:10.1186/s13058019-1241-x

14. Anonymous, "Breast cancer in Australia statistics," 2015Available: https://breast-cancer.canceraustralia.gov.au/statistics. Accessed August $12,2020$.

15. Arjonen A, Kaukonen R, Ivaska J. Filopodia and adhesion in cancer cell motility. Cell Adhes Migr. 2011;5(5):421-430. doi:10.4161/ cam.5.5.17723

16. Jiang P, Enomoto A, Takahashi M. Cell biology of the movement of breast cancer cells: intracellular signalling and the actin cytoskeleton. Cancer Lett. 2019;284(2):122-130. doi:10.1016/j.canlet.2009.02.034

17. Khan H, Ullah H, Martorell M, et al. Flavonoids nanoparticles in cancer: treatment, prevention and clinical prospects. Semin Cancer Biol. 2019. doi:10.1016/j.semcancer.2019.07.023

18. Sayed N, Khurana A, Godugu C. Pharmaceutical perspective on the translational hurdles of phytoconstituents and strategies to overcome. J Drug Deliv Sci Technol. 2019;53:101201. doi:10.1016/j.jddst.20 19.101201

19. Lee IC, Choi BY. Withaferin-A-A Natural anticancer agent with pleitropic mechanisms of action. Int J Mol Sci. 2016;17:3. doi:10.3390/ijms 17030290

20. Dhar N, Razdan S, S R, Bhat WW, Vishwakarma R, Lattoo SK. A decade of molecular understanding of withanolide biosynthesis and in vitro studies in withania somnifera (1.) dunal: prospects and perspectives for pathway engineering. Front Plant Sci. 2015;6. doi: 10.3389/fpls.2015.01031

21. Dey A, Chatterjee SS, Kumar V. Triethylene glycol-like effects of Ashwagandha (Withania somnifera (L.) Dunal) root extract devoid of withanolides in stressed mice. AYU Int $Q J$ Res Ayurveda. 2018;39 (4). doi:10.4103/ayu.AYU_219_16

22. Thipe VC, Amiri KP, Bloebaum P, et al. Development of resveratrol-conjugated gold nanoparticles: interrelationship of increased resveratrol corona on anti-tumor efficacy against breast, pancreatic and prostate cancers. Int $J$ Nanomedicine. 2019;14:4413-4428. doi:10.2147/IJN.S204443

23. Khan T, Gurav P. PhytoNanotechnology: enhancing Delivery of Plant Based Anti-Cancer Drugs. Front Pharmacol. 2018;8. doi:10.3389/ fphar.2017.01002

24. Martínez-Ballesta M, Gil-Izquierdo Á, García-Viguera C, Domínguez-Perles R. Nanoparticles and controlled delivery for bioactive compounds: outlining challenges for new 'smart-foods' for health. Foods. 2018;7(5):72. doi:10.3390/foods7050072 
25. Khoobchandani, M. Targeted phytochemical-conjugated gold nanoparticles in cancer treatment: environmental issues in logistics and manufacturing. Biotech Prod Everyday Life. 2019;37-52. doi:10.10 07/978-3-319-92399-4 3

26. Yadav DK, Kumar S, Saloni S, et al. Molecular docking, QSAR and ADMET studies of withanolide analogs against breast cancer. Drug Des Devel Ther. 2017;11:1859-1870. doi:10.2147/DDDT.S130601

27. Nile SH, Nile GE, Baskar V, Kai G, Kai G. Subcritical water extraction of withanosides and withanolides from ashwagandha (Withania somnifera L) and their biological activities. Food Chem Toxicol in $J$ Publ Br Ind Biol Re Assoc. 2019;132:110659. doi:10.1016/j. fct.2019.110659

28. Bennett HL, Brummer T, Jeanes A, Yap AS, Daly RJ. Gab2 and Src co-operate in human mammary epithelial cells to promote growth factor independence and disruption of acinar morphogenesis. Oncogene. 2008;27(19):2693-2704. doi:10.1038/sj.onc.1210928

29. Kimling J, Maier M, Okenve B, Kotaidis V, Ballot H, Plech A. Turkevich method for gold nanoparticle synthesis revisited. J Phys Chem. 2006;110(32):15700-15707. doi:10.1021/jp061667w

30. Frens G. Controlled Nucleation for the Regulation of the Particle Size in Monodisperse Gold Suspensions. Nat Phys Sci. 1973;241 (105):20-22. doi:10.1038/physci241020a0

31. Piella J, Bastús NG, Puntes V. Size-controlled synthesis of sub-10nanometer citrate-stabilized gold nanoparticles and related optical properties. Chem Mater. 2016;28(4):1066-1075. doi:10.1021/acs. chemmater.5b04406

32. Perrault SD, Chan WCW. Synthesis and surface modification of highly monodispersed, spherical gold nanoparticles of 50-200 nm. J Am Chem Soc. 2009;131(47):17042-17043. doi:10.1021/ja907069u

33. Trouiller AJ, et al. Biocompatible spherical gold nanoparticles synthesis in aqueous tetraethylene oxide solution and their cellular uptake. J of Nanosci. 2019. doi:10.1166/jnn.2019.16304
34. Romero-Benavides JC, Ortega-Torres GC, Villacis J, VivancoJaramillo SL, Galarza-Urgilés KI, Bailon-Moscoso N. Phytochemical Study and Evaluation of the Cytotoxic Properties of Methanolic Extract from Baccharis obtusifolia. Int J Med Chem. 2018;2018:1-5. doi:10.1155/2018/8908435

35. Aslan K, Pérez-Luna VH. Surface Modification of Colloidal Gold by Chemisorption of Alkanethiols in the Presence of a Nonionic Surfactant. Langmuir. 2002;18(16):6059-6065. doi:10.1021/la025 $795 x$

36. Rycenga M, Cobley CM, Zeng J, et al. Controlling the synthesis and assembly of silver nanostructures for plasmonic applications. Chem Rev. 2011;111(6):3669-3712. doi:10.1021/cr100275d

37. Shafiqa AR, Abdul Aziz A, Mehrdel B. Aziz AA and Mehrdel B. Nanoparticle optical properties: size dependence of a single gold spherical nanoparticle. J Phys Conf Ser. 2018;1083:012040. doi:10.1088/1742-6596/1083/1/012040

38. González ML, Joray MB, Laiolo J, et al. Cytotoxic Activity of Extracts from Plants of Central Argentina on Sensitive and Multidrug-Resistant Leukemia Cells: isolation of an Active Principle from Gaillardia megapotamica. Evid Based Complement Alter Med. 2018;2018:1-13. doi:doi.10.1155/2018/9185935

39. Cruz E, Kayser V. Synthesis and enhanced cellular uptake in vitro of anti-her2 multifunctional gold nanoparticles. Cancers. 2019;11(6).

40. Vijayakumar S. Eco-friendly synthesis of gold nanoparticles using fruit extracts and in vitro anticancer studies. J Saudi Chem Soc. 2019;23(6):753-761. doi:10.1016/j.jscs.2018.12.002

41. Agarwalla P, Mukherjee S, Sreedhar B, Banerjee R. Glucocorticoid receptor-mediated delivery of nano gold-withaferin conjugates for reversal of epithelial-to-mesenchymal transition and tumor regression. Nanomed. 2016;11(19):2529-2546. doi:10.2217/nnm2016-0224
International Journal of Nanomedicine

\section{Publish your work in this journal}

The International Journal of Nanomedicine is an international, peerreviewed journal focusing on the application of nanotechnology in diagnostics, therapeutics, and drug delivery systems throughout the biomedical field. This journal is indexed on PubMed Central, MedLine, CAS, SciSearch ${ }^{\mathbb{}}$, Current Contents ${ }^{\mathbb{R}} /$ Clinical Medicine, $^{-}$

\section{Dovepress}

Journal Citation Reports/Science Edition, EMBase, Scopus and the Elsevier Bibliographic databases. The manuscript management system is completely online and includes a very quick and fair peer-review system, which is all easy to use. Visit http://www.dovepress.com/ testimonials.php to read real quotes from published authors. 